\title{
First- and Second-Order Thermal Diffuse Scattering (TDS) Intensity in Molecular Crystals: Influence on Crystal Structure Parameters
}

\author{
By A. Criado, A. Conde and R. Márquez \\ Departamento de Optica y Sección de Física Centro Coordinado del CSIC, Universidad de Sevilla, Spain
}

(Received 24 July 1984; accepted 22 April 1985)

\begin{abstract}
First- and second-order thermal diffuse scattering (TDS) intensities are calculated in the long-wave approximation allowing for dispersion (LWD) in monoclinic phenothiazine from polarization vectors and lattice-mode frequencies obtained from lattice dynamical calculations within the harmonic approximation and the external Born-von Kármán formalism using an atom-atom potential function in the form $V(r)=-A / r^{6}+B \exp (-C r)$. The influence of firstand second-order TDS intensity on electronic density maps is analysed and compared. Least-squares refinements of positional and thermal parameters are carried out in different ranges of $\sin \theta / \lambda$ taking into account both first- and second-order TDS contributions and the results are discussed.
\end{abstract}

\section{Introduction}

In previous papers (Criado, Conde \& Márquez, $1985 a, b)$ we reported a computational procedure to calculate first- and second-order thermal diffuse scattering intensity for molecular crystals from a lattice dynamical calculation, using the external Born-von Kármán formalism in the harmonic approximation. An atom-atom potential-function model was utilized as a sum of pairwise contributions in the form $V(r)=$ $-A / r^{6}+B \exp (-C r)$ and the procedure was applied to monoclinic phenothiazine. First-order correction factors to integrated Bragg intensities due to thermal diffuse scattering (TDS) intensity were calculated with the full lattice dynamical formulae and in the long-wave (LWD) (Born \& Huang, 1968) approximation allowing for dispersion and both results were practically identical. The influence of first-order TDS intensity on electronic density maps was studied; the main contribution was a positive additional density concentration around the atomic positions. A leastsquares process was carried out to check the influence of first-order TDS intensity upon variable parameters; the main influence was found over the thermal parameters, which underwent a decrease with respect to their true value.

Calculations have already been performed (Helmoldt \& Vos, 1977) on the influence of the first-

0108-7673/85/050491-04\$01.50 order TDS contribution upon structural parameters and our purpose here is to study the influence of the second-order one.

\section{Basic theory}

First-order TDS intensity has been calculated using the LWD approximation in which the expression of the intensity at a point $\mathbf{S}$ of the reciprocal space such that $\mathbf{S}=\mathbf{G}-\mathbf{q}$, where $\mathbf{G}=2 \pi \mathbf{H}$ and $\mathbf{H}$ is a reciprocallattice point, is given by (Cochran, 1963; Cochran \& Pawley, 1964; Born \& Huang, 1968)

$$
\begin{aligned}
& \mathrm{d} I_{1}(\mathbf{S}=\mathbf{G}-\mathbf{q}) / \mathrm{d} \mathbf{q} \\
& \quad=N S^{2}|F(\mathbf{G})|^{2} \sum_{j_{\mathrm{ac}}}\left[E_{j}(\mathbf{q}) / \omega_{j}^{2}(\mathbf{q})\right]\left\{\mathbf{s} \cdot \mathrm{e}^{\mathrm{Lw}}(\mathbf{q} j)\right\}^{2},
\end{aligned}
$$

where $N$ is the number of unit cells in the crystal, $j_{\mathrm{ac}}$ stands for the different acoustic modes with wave vector $\mathbf{q}, \omega_{j}(\mathbf{q})$ is the angular frequency of mode $(\mathbf{q} j)$, $E_{j}(\mathbf{q})$ is its energy, $\mathbf{s}$ is a unit vector along $\mathbf{S}, F(\mathbf{G})$ is the Bragg structure factor and $\mathrm{e}^{\mathrm{LW}}(\mathbf{q} j)$ is the polarization vector of mode $(q j)$ in the LW approximation; it can be found by solving the eigenvalue equation (Born \& Huang, 1968; Maradudin, Montroll, Weiss \& Ipatova, 1971)

$$
D(\mathbf{q}) \mathbf{U}(\mathbf{q})=\omega^{2}(\mathbf{q}) \mathbf{U}(\mathbf{q}),
$$

where $D(\mathbf{q})$ is the dynamical matrix.

The second-order TDS intensity should be calculated in the exact lattice dynamical procedure but computing times are prohibitive. Because of this, we have also adopted the LWD approximation for calculating second-order TDS intensity. With this approximation, the expression for it is

$$
\begin{aligned}
& \mathrm{d} I_{2}(\mathbf{S}=\mathbf{G}-\mathbf{q}) / \mathrm{dq} \\
& =\left(S^{2} / 2\right)|F(\mathbf{G})|^{2} \sum_{\mathbf{q}^{\prime}} \sum_{j_{\mathrm{ac}}^{\prime}} \sum_{j_{\mathrm{ac}}^{\prime \prime}}\left\{\left[E_{j^{\prime}}\left(\mathbf{q}^{\prime}\right) E_{j^{\prime \prime}}\left(\mathbf{q}^{\prime \prime}\right) / \omega_{j^{\prime}}^{2}\left(\mathbf{q}^{\prime}\right) \omega_{j^{\prime \prime}}^{2}\left(\mathbf{q}^{\prime \prime}\right)\right]\right. \\
& \left.\quad \times\left[\mathbf{s} \cdot \mathbf{e}^{\mathrm{LW}}\left(\mathbf{q}^{\prime} j^{\prime}\right) \mathbf{s} \cdot \mathbf{e}^{\mathrm{LW}}\left(\mathbf{q}^{\prime \prime} j^{\prime \prime}\right)\right]^{2}\right\}
\end{aligned}
$$

where $q^{\prime}$ runs over all the allowed wave vectors inside the Brillouin zone and $\mathbf{q}=\mathbf{q}^{\prime}+\mathbf{q}^{\prime \prime}$. This sum takes a long computing time for a crystal of a finite size and, as we are only interested in the behaviour of density maps and structural parameters with respect to the

(C) 1985 International Union of Crystallography 
factor $S^{4}$, we have adopted a second simplifying approximation (Ramachandran \& Wooster, 1951), which consists of a theoretical evaluation of the sum (integral for a large crystal) over $\mathbf{q}^{\prime}$ assuming a linear relation $\omega_{j}(\mathbf{q})=v_{j} q$ (LW approximation), where $v_{j}$ is the velocity of the wave, which is assumed to be independent of the direction of $q$. The factor involving the polarization vectors is replaced with a constant factor at the values that minimize the product $\omega_{j^{\prime}}^{2}\left(\mathbf{q}^{\prime}\right) \omega_{j^{\prime \prime}}^{2}\left(\mathbf{q}^{\prime \prime}\right): \mathbf{q}^{\prime}=\mathbf{q}^{\prime \prime}=\mathbf{q} / 2$; and the integral over the Brillouin zone is extended to infinity. With these approximations, the expression of second-order TDS intensity for points sufficiently near the reciprocallattice points is found to be, at high temperatures $\left[E_{j}(\mathbf{q})=K_{B} T\right]$ :

$$
\begin{aligned}
& \mathrm{d} I_{\mathbf{2}}(\mathbf{S}=\mathbf{G}-\mathbf{q}) / \mathrm{dq} \\
& =\left(N V_{c} S^{4} q^{3} / 16\right)\left(K_{B} T\right)^{2}|F(\mathbf{G})|^{2} \\
& \times \sum_{j_{\text {ac }}^{\prime} \sum_{\text {ac }}}\left\{\left[\mathbf{s} . \mathbf{e}^{\mathrm{LW}}\left(\mathbf{q} j^{\prime}\right) \mathbf{s} \cdot \mathbf{e}^{\mathrm{LW}}\left(\mathbf{q} j^{\prime \prime}\right)\right]^{2} / \omega_{j^{\prime}}^{2}(\mathbf{q}) \omega_{j^{\prime \prime}}^{2}(\mathbf{q})\right\} \text {, }
\end{aligned}
$$

where $V_{c}$ is the unit-cell volume.

\section{Method of calculation}

We have performed our calculations with monoclinic phenothiazine and the conditions for calculating the correction factors of Bragg intensities due to TDS contribution were the same as those adopted for first-order calculations (Criado, Conde \& Márquez, $1985 a$ ), assuming a Bragg-peak symmetric scanning volume of parallelepipedic shape centred on the reciprocal-lattice points, each edge $7 / 25$ of the corresponding basic reciprocal vector. Polarization vectors and wave frequencies have been obtained from lattice dynamical calculations.

In order to study the influence that second-order TDS intensity has over density maps we have considered the crystal configuration from which we have calculated lattice dynamics and Bragg correction factors as the true one. The intensities measured in a real experiment would then be obtained by adding to the calculated Bragg intensities the TDS calculated contribution at $300 \mathrm{~K}$ as

$$
I_{\text {exp }}(\mathbf{G})=I_{\text {BRAGG }}(\mathbf{G})\left\{1+\alpha_{2}(\mathbf{G})\right\},
$$

where

$$
\alpha_{2}(\mathbf{G})=I_{2}(\mathrm{TDS}, \mathbf{G}) / I_{\mathrm{BRAGG}}(\mathbf{G}),
$$

and the influence on density maps would be obtained by means of a difference Fourier synthesis with coefficients $F_{\text {exp }}(\mathbf{G})-F_{\text {cal }}(\mathbf{G})$.

In this way we have calculated correction factors and 'experimental' intensities for 1026 independent reflections with $\sin \theta / \lambda<0.6 \AA^{-1}$. A difference Fourier synthesis (program FOURR; Stewart, Kundell \& Baldwin, 1970) has been performed, whose

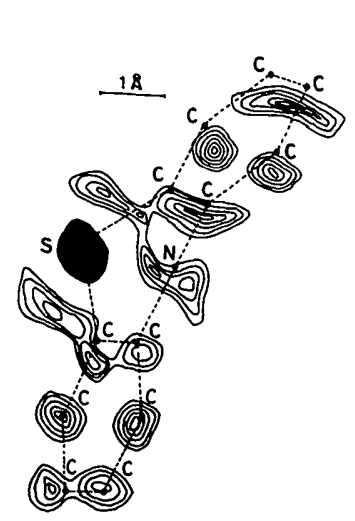

(a)

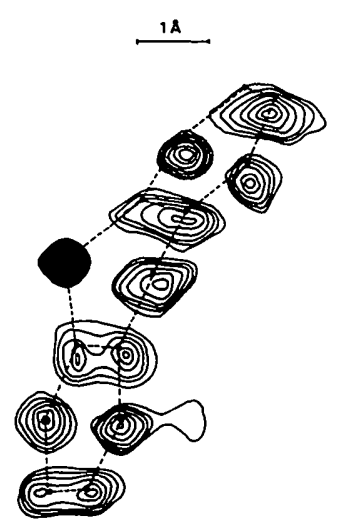

(b)
Fig. 1. (a) Second-order difference density map. Contours are drawn at intervals of $0.012 \mathrm{e}^{-2}$. (b) First-order difference density map. Contours are drawn at intervals of $0.06 \mathrm{e} \AA^{-2}$.

projection over a plane perpendicular to $\mathbf{b}$ is shown in Fig. 1 $(a)$, where only positive density regions are represented, and the black points correspond to the 'true' atomic positions. In order to make the comparison easier we reproduce in Fig. $1(b)$ the difference density map obtained with the same reflections but considering only the first-order contribution (Criado, Conde \& Márquez, 1985a). Both maps present a zone of negative density surrounding each positive peak, a result that is found to correspond to an undervaluation of thermal crystallographic parameters (Buerger, 1960). As in the first-order case, positive density peaks in the second-order map are situated around the 'true' atomic positions and therefore it must be expected that the second-order TDS contribution will not alter very much the positional parameters obtained in a structural analysis. Nevertheless, distortions of the peaks are greater than in the firstorder case, especially near the heavier atom (S) indicating that the second-order TDS effect is more equally distributed between positional and thermal parameters than in the first-order case. Whereas in the first-order map peaks around $\mathrm{H}$ atoms are practically non-existent, we have detected small peaks near $\mathrm{H}$-atom positions, which may well correspond to an alteration of the $\mathrm{H}$-atom density, although they may be a residual electronic density as well.

\section{Least-squares refinement}

To verify the conclusions deduced above we have carried out a least-squares process (program CRYLSQ; Stewart, Kundell \& Baldwin, 1970) with the 'experimental' intensities at $300 \mathrm{~K}$ from the 'true' structure and calculated lattice dynamical thermal parameters, keeping the thermal parameters of $\mathrm{H}$ atoms fixed. Positional and thermal parameters were refined together with $K$, a scale factor defined by $\sum_{\mathbf{G}}\left\{\left|F_{\text {exp }}(\mathbf{G})\right|-\left|F_{\text {cal }}(\mathbf{G})\right| / K\right\}^{2}$, and three different 


\section{Table 1. Results of least-squares refinements}

f.a. full angle; 1.o. low order; h.o. high order.

$\Delta \mathbf{r}$ and $\Delta U_{i i}$ are the variations in positional and thermal parameters.

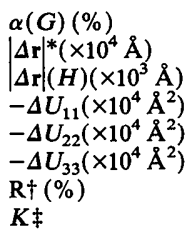

First order

\begin{tabular}{ccc}
\hline f.a. & 1.0. & h.o. \\
$<80$ & $<37$ & $37-107$ \\
$5-10$ & $4-8$ & $11-18$ \\
$5-8$ & $3-6$ & $18-38$ \\
$55-57$ & $56-58$ & $48-50$ \\
$51-55$ & $52-54$ & $44-46$ \\
$47-51$ & $48-51$ & $41-45$ \\
$8 \cdot 4-0.4$ & $6 \cdot 4-0 \cdot 3$ & $21 \cdot 4-0 \cdot 2$ \\
$1 \cdot 005$ & $1 \cdot 003$ & $1 \cdot 047$
\end{tabular}

* Average and maximum deviations.

$\dagger$ Initial and final agreement factors

$\ddagger$ Final scale factor. ranges have been chosen with 1026 reflections in each one: a full-angle refinement up to $\sin \theta / \lambda=0.9 \AA^{-1}$, a low-order refinement up to $\sin \theta / \lambda=0.6 \AA^{-1}$ and a high-order one with $0.7<\sin \theta / \lambda<1.0 \AA^{-1}$. A similar process has been performed with first-order contributions and we compare the main results in Table 1. Variations in positional parameters of non- $\mathrm{H}$ atoms are of the same order of accuracy in crystallographic work and are alike for first and second order in spite of the smaller magnitude of second-order correction factors. On the contrary, variations for $\mathrm{H}$-atom coordinates are greater for second-order refinements, a result that agrees with the conclusions drawn from the difference Fourier maps.

Thermal parameters present a decrease for both first- and second-order refinements. For first order the parameters are smaller at high angles because correction factors are large and $1+\alpha_{1}(\mathbf{G})$ cannot be adjusted to a temperature factor with exponential form as well as in full angle or low-order cases. Maximum deviations for thermal parameters are very close to average, indicating that the effect is more important for the translational rigid-body tensor $\mathbf{T}$ than the librational $\mathbf{L}$. This seems to confirm the proposal from the recent project report on the comparison of structural parameters for oxalic acid dihydrate obtained in various laboratories (Coppens 1984), which shows the main discrepancies are in thermal parameters, principally on $\mathbf{T}$ tensors, which are probably due to TDS contributions. The nontransferability of thermal parameters from high-order to low-order refinement must be taken into account when calculating thermal parameters in accurate electronic density studies (Dam, Harkema \& Feil, 1983) from reflections at high values of $\sin \theta / \lambda$ where the influence of bonding effects over thermal parameters is minimized when we use a spherical-atom model.

In the case of second-order refinements we obtain different values of thermal parameters depending on the chosen range of $\sin \theta / \lambda$ because $1+\alpha_{2}(\mathbf{G})$ does not adjust at all to a temperature-factor functional form, even at low angles; and the pattern is the inverse to the first-order case: the decrease is larger in highorder refinements. In the same way, variations in the scale factor are opposite in first- and second-order cases.

\section{Concluding remarks}

Positional parameters are more sensitive to secondthan to first-order TDS intensity, especially those concerning hydrogen atoms. Thermal parameters are different for each range when the second-order TDS contribution is not subtracted from measurements and, although its influence is small for low-angle reflections, it is comparable to that of first-order for high-order refinements. So experimental intensities should be corrected for second-order contributions when calculating accurate thermal parameters from high-order refinements, although the best remedy is to work at as low a temperature as possible.

Final agreement factors $R$ are equal or larger for second-order refinements, although initial factors are much smaller than first-order ones, indicating a poorer absorption of TDS contributions in leastsquares processes.

As a final overall conclusion we can say that even when the second-order contribution is smaller than the first-order one, its effects may be comparable to first-order ones and are more equally distributed among different variable parameters and are not predominantly concentrated on thermal parameters, which is the case for the first-order contribution.

This work has been supported in part by the Spanish Government through the "Comisión Asesora de Investigación Científica y Técnica'.

\section{References}

Born, N. \& HuANG, K. (1968). Dynamical Theory of Crystal Lattices. Oxford: Clarendon.

BUERger, M. J. (1960). Crystal-Structure Analysis. New York: Wiley. 
Cochran, W. (1963). Rep. Prog. Phys. 26, 1-45.

Cochran, W. \& Pawley, G. S. (1964). Proc. R. Soc. London, 280, 1-22.

Coppens, P. (Project Reporter) (1984). Acta Cryst. A40, 184-195.

Criado, A., Conde, A. \& Márquez, R. (1985a). Acta Cryst. A41, 158-163.

Criado, A., Conde, A. \& Márquez, R. (1985b). Acta Cryst. A41, 316-320.

DAM, J., HaRkema, S. \& Feil, D. (1983). Acta Cryst. B39, 760-768.
Helmholdt, R. B. \& Vos, A. (1977). Acta Cryst. A33, 38-45.

Maradudin, A. A., Montroll, E. W., Weiss, G. H. \& IPATOVA, I. P. (1971). Theory of Lattice Dynamics in the Harmonic Approximation. New York: Academic Press.

RAMAChandRan, G. N. \& Wooster, W. A. (1951). Acta Cryst. 4, 335-344.

Stewart, J. M., Kundell, F. A. \& Baldwin, J. C. (1970). The XRAY 70 system. Computer Science Center, Univ. of Maryland, College Park, Maryland.

Acta Cryst. (1985). A41, 494-500

\title{
Calculation of Elastic Constants by the Method of Crystal Static Deformation*
}

\author{
By Michele Catti \\ Dipartimento di Chimica Fisica ed Elettrochimica, Università di Milano, via Golgi 19, 20133 Milano, Italy
}

(Received 7 November 1984; accepted 22 April 1985)

\begin{abstract}
The contribution of homogeneous lattice deformations (neglecting internal strains) to elastic properties of crystals with triclinic or higher symmetry is examined. The deformed lattice constants are expressed as functions of the components of the finite Lagrangian strain tensor, and their derivatives are calculated. Thus equations are obtained that relate the second-order elastic constants to first and second partial derivatives of the static crystal energy with respect to unit-cell parameters. With the assumption of a two-body Born-type interatomic potential, the energy derivatives were calculated analytically, and a rigid-body approximation was introduced to account for molecular groups in the crystal structure. Test computations of elastic constants were performed for $\mathrm{MgF}_{2}$ (rutile-type), benzene and naphthalene, using literature potential parameters optimized on structural data; results are discussed with respect to adequacy of the potentials and of the approximations of the model used.
\end{abstract}

\section{Introduction}

The semi-empirical modelling of interatomic and intermolecular forces in crystals has been developed intensively in recent years, in order to reproduce and possibly predict various chemical-physical properties by computer simulations (Catlow \& Mackrodt, 1982). In the past, this work was mainly performed by fitting

* A preliminary account of part of this work was presented at the XIIIth International Congress of Crystallography, Hamburg, Federal Republic of Germany, 9-18 August 1984 (Catti, 1984). the potential parameters to structural properties only, so that the least-energy atomic configuration approached the experimental one as closely as possible (Busing, 1970; Kitaigorodskii, 1973; Williams, 1981); such potentials were then proposed for determining unknown crystal structures by minimumenergy search. In attempts to extend the modelling to other physical properties of crystals, elastic behaviour and vibrational spectroscopic frequencies are usually considered, as they are related to the slope changes of the energy hypersurface (in the space of atomic position vectors) at its minimum point. However, vibrational properties are accounted for by dynamical methods only, whereas the crystal elasticity can be related both to lattice dynamics and to the statics of equilibrium atomic configuration. In the former case a microscopic crystal deformation changing with time is examined, through atomic oscillations during the propagation of an elastic wave (longwavelength acoustic vibration mode); in the latter, a macroscopic static deformation of the crystal is assumed, implying atomic shifts from equilibrium positions that are constant with time. In both cases the elastic properties express the correlation between crystal strain and applied stress. The first full theory on the subject was developed by Born \& Huang (1954).

A previous partial approach (Catti, 1981) is extended and the calculation of elastic constants by the method of crystal static deformation is considered here. The contribution of external strains will be taken into account by deriving equations that relate the elasticity tensor components to first and second partial derivatives of the crystal static energy with respect to lattice constants, calculated at zero strain, for tri-

(C) 1985 International Union of Crystallography 